BULLETIN (New Series) OF THE

AMERICAN MATHEMATICAL SOCIETY

Volume 48, Number 2, April 2011, Pages 229-257

S 0273-0979(2010)01319-7

Article electronically published on December 2, 2010

\title{
CRYSTALS, PROTEINS, STABILITY AND ISOPERIMETRY
}

\author{
MISHA GROMOV
}

\begin{abstract}
We attempt to formulate several mathematical problems suggested by structural patterns present in biomolecular assemblies. Our description of these patterns, by necessity brief and over-concentrated in some places, is self-contained, albeit on a superficial level. An attentive reader is likely to stumble upon a cryptic line here and there; however, things will become more transparent at a second reading and/or at a later point in the article.
\end{abstract}

\section{Contents}

1. Is there mathematics in biology? 229

2. Periodic molecular assemblies 231

3. Crystals in fluids: erythrocytes, liposomes, micelles 235

4. Information and symmetry in viruses 240

5. Polypeptides and proteins: sequences, folds, and functions 242

6. Energy landscapes and protein problems 249

About the author 255

References 256

\section{IS THERE MATHEMATICS IN BIOLOGY?}

Day-Night, Day-Night, Day-Night. Summer-Winter, Summer-Winter....

What is the structure behind this? Where should we look to uncover it? Is it in the singing of birds, in the flows of rivers, in the changes of temperature?

We know, with hindsight, what we should do.

Look up into the night sky, locate a few specks of light - planets - that slowly crawl amongst the relatively unmoving stars. We imagine how their routes appear if seen from the sun, invent calculus, guess differential equations for the planetary routes, unravel symmetries of these,.... and the world of mathematical wonders opens to you: Lie groups, algebraic varieties, symplectic manifolds....

Now try: Alive-Dead, Alive-Dead, Alive-Dead.... Where do we go from here?

"... living matter, while not eluding the 'laws of physics' as established up to date, is likely to involve 'other laws of physics' hitherto unknown, which however, once they have been revealed, will form just as integral a part of science as the former".

Received by the editors September 24, 2009, and, in revised form, May 10, 2010.

2010 Mathematics Subject Classification. Primary 92-XX; Secondary 58-XX, 53-XX. 
Erwin Schrödinger, who wrote this in 1944 in his book What Is Life?, apparently had in mind some counterpart to the Second Law of Thermodynamics. The "other laws of physics" have not materialized. But biology has gone far since 1944 - the molecular patterns of Life have been displayed before our eyes as the stars in the night sky.

But what are the specs of light that would guide us to the world of new mathematics?

At first sight, Nature does not appear exceptionally clever: her evolutionary strategy is not sophisticated, to say the least. But she was selecting from billions upon billions of candidates and her selection criterion, "fit to survive", may look simple only for a lack of mathematical imagination on our part: an enormous amount of structure goes into this "fit". Besides, Nature does not run in a structural vacuum - all of physics and chemistry is at her disposal, and she excels in molecular dynamics and in catalysis.

Yet, a mathematician might think that Nature is dumb: the primitive mutation/selection mechanism of evolution could not produce anything we, mathematicians, could not divine ourselves.

But if so, we inevitably conclude that the human brain, which was cooked up by Nature in the last couple of million years, cannot be especially smart either: all our mathematics, or rather the mathematics-building mechanisms in the brain, must be confined to the rules that evolution had stumbled upon in this relatively short stretch of time and had installed into us.

On the other hand, Nature had spent a much longer time (measured by the number of tries involved) in inventing such structural entities as the cell and the ribosome.

(Ribosomes are large molecular assemblies $\approx 25 \mathrm{~nm}=2.5 \cdot 10^{-6} \mathrm{~cm}$ in diameter composed of ribosomal RNA and proteins. As a ribosome crawls along a messenger RNA it synthesizes a polypeptide chain out of $20(+1)$ amino acids in the cell, 1020 residues per second, by translating genetic information written on this RNA in four letters - four species of basic units - nucleotide molecules, where an RNA is a hundreds-/thousands-long polymer chain composed of these units.

There are usually many ribosomes translating in parallel from a single RNA molecule with $\approx 100$ or fewer nucleotides between them; one might say that it is RNA that crawls through a train of ribosomes.

One may conjecture that neither cell nor brain would be possible if not for profound mathematical "somethings" behind these, Nature's inventions. But what are these "somethings"? Why do we, mathematicians, remain unaware of them?

Notwithstanding our much-glorified successes, we are, tautologically, blind to what we do not see. (Nature systematically hides from our mind what we are not supposed to know, such as the blind spot in our retina, for instance. The neurological mechanism of this hiding is far from clear.)

Also, the history of mathematics shows how slow we are when it comes to inventing/recognizing new structures even if they are spread before our eyes, such as hyperbolic space, for instance. (More recent and more relevant examples are seen in the slow start in the mathematical development of Mendelian genetics and in the failure of identifying general mathematical principles underlying Sturtevant's reconstruction of the linear structure on the set of genes on a chromosome of Drosophila 
melanogaster from samples of a probability measure on the space of gene linkages [13.)

The brain is hardly able to generate mathematical concepts by itself; it needs an input of "raw structures", and Nature has much to offer. The problem is that this "much" which the biology offers to us is "too much": it is hard to decide what in this offering contains germs of new mathematics and what is a "frozen accident", an irrelevantly special complexity.

The only way to reject the irrelevant is to first learn and understand what it is. One has to browse through myriad stars - structural specks of Life revealed by biologists - in order to identify the "essential ones", and when (if?) we find them, we may start on the long road toward new mathematics.

And even if we fail to assemble a coherent structure from the multitude of available fragments, we may gain a better vision on the boundary of our mathematical knowledge which is hidden from us by the "complacency wall" of an intrinsically mathematical point of view.

\section{Periodic molecular assemblies}

The $\mathbb{Z}^{3}$-symmetry of crystals, i.e., the triple periodicity on the atomic/molecular scale, was discovered/conjectured by René Just Haüy in the late 1700s who, according to mineralogists' lore, came to the idea while pondering over the fragments of a broken calcite crystal. (His Traité de Minéralogie appeared in 1801.)

But why symmetry? It seems unlikely that if you shake potatoes in a big box they would spontaneously arrange themselves into something symmetric; yet, this happens to many large potato-shaped molecules, such as myoglobin (see Figure 2) a protein with $\approx 3 \mathrm{~nm}$ diameter molecules $\left(1 \mathrm{~nm}=10^{-3} \mu \mathrm{m}=10^{-7} \mathrm{~cm}=10^{-9} \mathrm{~m}\right)$ made of about 2,000 atoms $-2-3 \AA$-balls $(1 \AA=0.1 \mathrm{~nm})$. (Myoglobin stores oxygen in muscles; it contains a metal-organic heme group with an iron atom to which $\mathrm{O}_{2}$ binds.)

The 3-D structure of myoglobin was the first protein structure solved by John Kendrew in 1958 with $X$-ray diffraction analysis.

The diffraction of X-rays delivers only the amplitude of the Fourier transform of the electron density in a crystal. The periodicity of the crystal is crucial for the extraction of the information on individual molecules on the $\AA$-scale from the

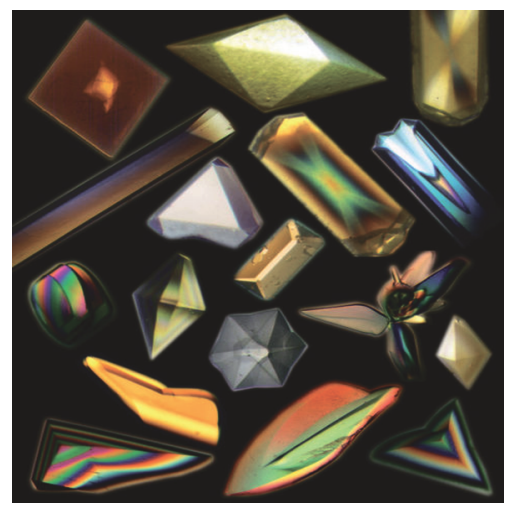

FIGURE 1. Protein crystals 


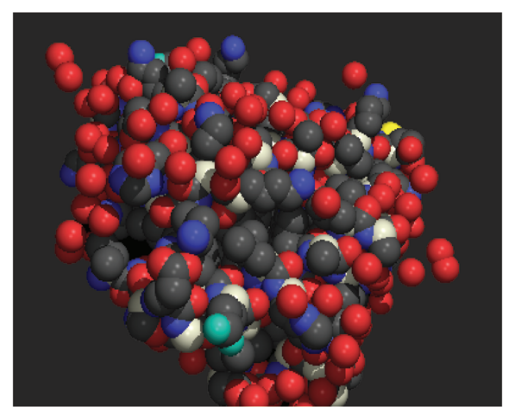

Figure 2. Myoglobin

diffraction image. This involves many nontrivial mathematical and nonmathematical ideas; see "X-ray crystallography" in Wikipedia.

How does the symmetry come about? The easiest to account for is the helical symmetry of molecular assemblies [8, 7], 21, 25]. Suppose that two molecular (sub)units of the same species $M$ preferentially bind by sticking (docking) one to another in a certain way (i.e., the binding energy for a pair of molecules has a unique minimum sufficiently separated from other local minima). If $M_{1} \vDash M_{2}$ is a pair of so bound molecules in the Euclidean space $\mathbb{R}^{3}$, then there is a (typically unique) isometric transformation (rigid motion) $T$ of $\mathbb{R}^{3}$ moving $M_{1}$ to $M_{2}$.

Such a $T$, by an elementary theorem, is made by a rotation around an axial line $L$ in $\mathbb{R}^{3}$ followed by a parallel translation along $L$. If the copies $M_{1}, M_{2}=$ $T\left(M_{1}\right), \ldots, M_{n}=T\left(M_{n-1}\right)$ do no overlap, the chain of $n$ copies of $M$, written as $M_{1} \vDash M_{2} \vDash \cdots \vDash M_{n}$, makes a helical shape in the space around $L$ which provides a minimum of the binding energy to the ensemble of $n$ molecules. This minimum, even if it is only a local one, has a rather large attraction basin (this needs a proof), which makes the helical arrangement kinetically quite probable.

Helical symmetries ( $\alpha$-helices) are ubiquitous in proteins as was determined by Pauling, Corey, and Branson in 1951 on the basis of the structures of amino acids and the planarity of peptide bonds between amino acids (residues) in proteins. (Another commonly present pattern in proteins, called a $\beta$-sheet, displays $\mathbb{Z} \oplus \mathbb{Z}$ symmetry; see section 5.)

DNA molecules also have helical symmetry. (Three types of DNA double helices have been found.) A DNA helix is composed of two polymer chains held together by hydrogen bonds, where each chain is composed, from four species of basic molecular units - nucleotides (Adenine, Guanine, Cytosine, and Thymine, with $15 \pm 1$ atoms in each of them), similar to the molecular units making RNA.

The DNA chains in cells are quite long and their spacial conformations are far from metric $\mathbb{Z}$-symmetry, even if the pattern of bonds in DNA is strictly $\mathbb{Z}$-periodic, due to a certain flexibility of the bonds similar to that of $\alpha$-helices. (E.g., human DNA $\approx 2.5 \cdot 10^{8}$ base pairs, which would be almost $10 \mathrm{~cm}$ in length if fully stretched, is packed into $\mathrm{a} \approx 10 \mu \mathrm{m}$ chromosome in the cell.)

Another kind of helix, making a (rather rigid) rod-shaped viral particle about $300 \mathrm{~nm}\left(=3 \cdot 10^{-5} \mathrm{~cm}\right)$ long and $15-20 \mathrm{~nm}$ in diameter, is that of the Tobacco mosaic virus capsid (exterior shell; see Figure 3 ) made from 2130 molecules of coat protein. (Imagine how one gets this "2130" or look into [17, [3], 32, [19.) 


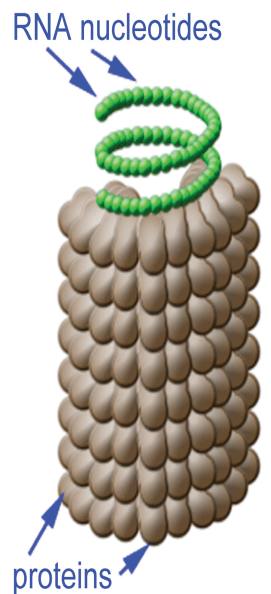

Figure 3. Tobacco mosaic virus

- Dmitri Ivanovski in 1892 provided evidence for a nonbacterial agent affecting tobacco plants which remained infectious after fine filtering.

- Wendell Stanley isolated and crystallized the virus in 1935 and showed that it remains active after crystallization.

- Gustav Kausche, Edgar Pfankuch, and Helmut Ruska obtained the electron microscopical images of the virus in 1939.

- James Watson studied the X-ray diffraction on the virus crystals in 19521954 and deduced its helical structure.

- Heinz Fraenkel-Conrat and Robley Williams showed in 1955 that purified viral RNA and its capsid protein assemble by themselves to functional viruses.

The biological helices, are more symmetric than the naked helix -(almost) every subunit has three or more neighbors bound to it. The origin of bio-helical and nonhelical symmetries can be understood by looking at the 2-dimensional version of the rigid motion $T$ in 3 -space.

A typical isometry $T$ of the plane is a rotation by some angle $\alpha$ around a fixed point, where this $\alpha$ is determined by the binding angle of $\vDash$ between $M_{1}$ and $M_{2}$. If $\alpha=2 \pi / n$ where $n$ is an integer, then the $n$ copies of $M$ make a roughly circular shape with the $n$-fold rotational symmetry.

Thus, for example, not all molecular units $M$ could form a 5 -fold symmetric assembly, but if the $\vDash$-angle is (planar and) close to $2 \pi / 5$ and if the $\vDash-$-bond is slightly flexible, then such an assembly, with every copy of $M$ involved into two "slightly bent" $\vDash-b o n d s$, will be possible. (Probably, there are several quasi-classical models of bending of a quantum covalent bond.)

Returning to the Euclidean 3 -space $\mathbb{R}^{3}$, if one wants a sufficiently rigid molecular assembly (e.g., a viral shell) $V$, where there are more than two, say four, neighbors for each copy of $M$ with two different kinds of $\vDash$ bonds and such that $V$ admits, say, two symmetries $T$ and $T^{\prime}$, then one needs to satisfy certain relations between the $\vDash-$ angles of mutually bound molecules, similar to but more complicated than the $2 \pi / n$-condition. 
These geometric relations must ensure the algebraic relations between the generators $T$ and $T^{\prime}$ in the expected symmetry group $\Gamma$, where $\Gamma$ is not given beforehandit comes along with the self-assembly process and may depend on specific kinetics. For example, the symmetry of protein crystals, one of 230 possible crystallographic groups, may depend on the particular condition at which a given protein is being crystallized (see "crystal structure" in Wikipedia and [16]).

The isometry group $\Gamma$ itself does not determine the geometry of a $\Gamma$-symmetric assembly: specific generators $T, T^{\prime}, \ldots$ of $\Gamma$ are essential. For example, the helical symmetry is governed by the group $\Gamma=\left\{\ldots, T^{-2}, T^{-1}, T^{0}=1, T^{1}, T^{2}, \ldots\right\}$, which is isomorphic to $\mathbb{Z}$ - the additive group of integers. This $\mathbb{Z}$, in biological helices, is given by two or more generators, say, by $T$ and $T^{\prime}$ with the relation $T^{\prime}=T^{n}$ with a moderately large $n$, e.g., $n=4$ for $\alpha$-helices, where $T$ is associated with the (strong covalent) peptide bonds and $T^{\prime}$ with (weak) hydrogen bonds between amino acid residues in a protein. (Sometimes $n=3$ and rarely $n=5$ in the $\alpha$-helices.) The Tobacco mosaic virus capsid has $T^{\prime}=T^{16}$ and $T^{\prime \prime}=T^{17}$. (Virologists represent this in different terms, e.g., in [2, 19, 3]; hopefully, my interpretation is correct.)

Alternatively, let us think in terms of the full configuration space $\mathcal{M}$ of molecules $M$, where there is an action $A$ or a family of actions, by a group $\Gamma$ on $\mathcal{M}$. If an (energy) function $E$ on $\mathcal{M}$ is invariant under these actions, then one can easily show in many cases that the local minima of $E$ on the subspace of $\Gamma$-symmetric configurations also serve as local minima on all of $\mathcal{M}$.

For example, $\mathbb{R}^{3}$ admits a 9 -parameter family of actions $A$ by the group $\Gamma=$ $\mathbb{Z}^{3}=\mathbb{Z} \oplus \mathbb{Z} \oplus \mathbb{Z}$ (where each $A$ is generated by three parallel translations) which induces in an obvious way actions $A$ of $\Gamma$ on the (infinite-dimensional) configuration space $\mathcal{M}$ of molecules in $\mathbb{R}^{3}$. Since the (mean) binding energy between molecules is invariant under $A$, the appearance of organic (triperiodic) crystals (regarded as minima points in the configuration space of molecules) looks less miraculous, even though the original group acting on the space $\mathbb{R}^{3}$ is its full isometry group, where a specific discrete subgroup $\Gamma$ arises in the process of a particular molecular assembly.

Crystals (and more general spatially correlated patterns 24]) have been much studied by physicists, but I doubt that a comprehensive purely mathematical model, incorporating an evaluation of the attraction basin of a crystal and a rigorous quantitative kinetics of crystallization, is available at the present day. Here are some questions.

How much does a symmetry of a molecule $M$ with a finite group $G$, which lies in the rotational quotient of some crystallographic group $\Gamma$, enhance the attraction basin of $\Gamma$-crystals of $M$ ?

How does a mixture of several different kinds of molecules crystallize?

For instance, protein crystals retain about $30 \%$ of water molecules which play an essential role in crystal formation [27.

One may think (on the basis of counting parameters) that a mixture, say, of $M, M^{\prime}$, and $M^{\prime \prime}$ with a random proportion $c: c^{\prime}: c^{\prime \prime}$ of the concentrations, would not crystallize, but there are particular $c: c^{\prime}: c^{\prime \prime}$ that are highly beneficial for crystallization, especially if $M^{\prime}$ and $M^{\prime \prime}$ are smaller than $M$ and nicely fit into the gaps between the $M$-molecules. (I guess, everything of this kind is known to crystallographers [28, but, in the long run, mathematicians may come up with something practically useful.) 
Forgetting physical crystals, a mathematician may wonder if something such as discrete subgroups of adelic groups also come into play by a process of crystallization from some huge configuration space.

\section{CRystals in Fluids: ERYthrocytes, Liposomes, MiCElles}

An erythrocyte - a red blood cell, the carrier of haemoglobin - is a roughly rotationally symmetric cell, kind of a biconcave disk (thin near the center and thickest at the periphery) of diameter $6000-8000 \mathrm{~nm}$ and of thickness $1500-1800 \mathrm{~nm}$ (small by animal cell standards). The membrane (surface) of an erythrocyte is a $6-8 \mathrm{~nm}$ thick bilayer of rod-like (phospholipid) molecules oriented normally to the surface of the membrane with hydrophilic "heads" facing the exterior and the interior of a cell while the hydrophobic "tails" are buried inside the membrane. Such a construction behaves as an incredible (quasi-inertionless) 2D-fluid curved in 3-space: free to move within itself (preserving area) but resisting bending.

Erythrocytes' (idealized) shape (see Figure 4) is believed to be a solution of an isoperimetric problem. Being a (closed simply connected) surface $S$ in 3 -space with prescribed area and 3-volume, it is bounded in space, which minimizes the integrated squared curvature encoding the bending energy of $S$. (See [15] and "Elasticity of cell membranes" in Wikipedia.)

(A similar picture is seen in the spherically symmetric shapes of small drops of liquids, of micelles, and of liposomes; see Figure 5. Spheres "solve" the isoperimetric problem: they surround a given volume by a surface of minimal area.

This is attributed to Dido who, according to ancient Greek and Roman sources, had solved the $2 D$-isoperimetric problem in the course of founding her kingdom of Carthage in circa 900 BC. But some historians are doubtful that Dido was influenced by erythrocytes, that she was aware of broken symmetries of solutions to fully symmetric problems, and even that she could furnish a rigorous proof of the sphericity of the minimizer to area/volume $e^{\frac{2}{3}}$.)

The corresponding variational/isoperimetric problem is easy in the class of rotationally symmetric surfaces, but I doubt that the rotational symmetry of the extremal surface has been proved.

The above motivates the following geometric variational problem.

Let $X$ be a $C^{\infty}$-smooth $n$-manifold, let an integer $k$ be given, let $X^{\prime}$ denote the Grassmannian bundle of tangent $k$-planes in $X$, and let $X^{(r)}$ be defined by $X^{(r)}=\left(X^{(r-1)}\right)^{\prime}$.

If $X$ carries a $C^{\infty}$ Riemannian metric $g_{X}$, then $g_{X}$ and the $O(n)$-invariant metric $g_{G r}$ in the Grassmannian fiber $\operatorname{Gr}_{k}\left(\mathbb{R}^{n}\right)$ define, via the Levi-Cevita splitting of the tangent bundle $T\left(X^{\prime}\right)$, a family of metrics $g_{p_{0}, p_{1}}^{\prime}=p_{0} g_{X}+p_{1} g_{G r}$ on $X^{\prime}$, for all $p_{0}, p_{1}>0$.

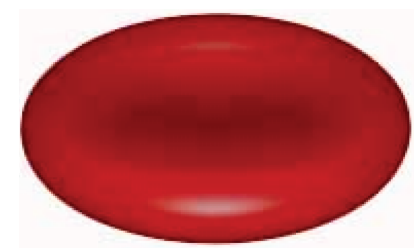

FIGURE 4. Erythrocyte 


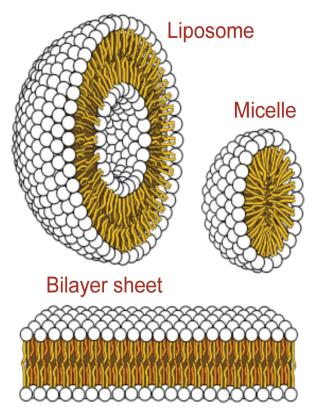

FiguRE 5. Liposome

Similarly, $X^{(r)}$ carries a family of metrics $g_{p}^{r}$ parametrized by the positive cone $P=\mathbb{R}_{+}^{r+1} \ni p$.

Every smooth $k$-submanifold $S \subset X$ lifts to $S^{(r)} \subset X^{(r)}$, and the $k$-volumes of these lifts with respect to $g_{p}^{r}$ define a function $\operatorname{vol}^{r}: \mathcal{S} \times P \rightarrow \mathbb{R}_{+}$, where $\mathcal{S}$ denotes the space of all $S$ and where $\mathrm{vol}^{r}$ is regarded as the family of functions $\operatorname{vol}_{p}^{r}$ on $\mathcal{S}=\mathcal{S} \times p$.

What are the critical points of $\operatorname{vol}_{p}^{r}$ ?

These only rarely belong to $\mathcal{S}$ itself; one needs to properly complete $\mathcal{S}$ (with respect to a suitable metric or otherwise) in order to allow certain singularities.

What is the (minimal) completion(s) for this? What are the singularities of extremal $S$ ?

Prominent examples of possibly singular vol $_{p}^{r}$-minimizers are complex subvarieties $S$ in algebraic/Kähler manifolds $X$. Probably (this seems easy) these $S$, even if singular, are stable as critical points under small non-Kählerian perturbations of the function $\operatorname{vol}_{p}^{r}$ (with suitably understood "stability" for nonisolated $S$ ).

How much does the function $\operatorname{vol}_{p}^{r}$ vary with $p$ on the subset of extremal $S$ ? In particular, how does it blow up (if at all) at the boundary of $P$ ?

How much does the picture change if we use another metric on $X^{(i+1)}$, e.g., associated to non-Levi-Civita connections on the bundles $X^{(i+1)} \rightarrow X^{(i)}$, for splitting the tangent bundles of $X^{(i+1)}$.

What is the symmetry of the solution(s) to the corresponding erythrocytes' isoperimetric $\mathrm{vol}^{r}$-problem for hypersurfaces in $\mathbb{R}^{n}$ for given $r$ and $n \gg r$.

What happens if, instead of the tangent $k$-planes, one uses tangent $l$-planes to $S$ for $l \neq k=\operatorname{dim} S$, e.g., for $l=n-1=\operatorname{dim} X-1$ on the first step?

The mathematics becomes more involved if we recall the physical origin of our surfaces $S$, which serve as boundaries of liposomes and, similarly, of micelles interfaces between water and hydrophobic substances (see "lipid bilayer" in Wikipedia). These emerge as (low temperature) limits of statistical ensembles $\mathcal{S E}$ (of many particle systems) of asymptotically infinite dimension, and the geometric PDE satisfied by such an $S$ (e.g., minimality, constant mean curvature, etc.) can be derived from the corresponding geometric properties of $\mathcal{S E}$.

(It is tempting to model such ensembles in the algebraic/Kählerian case by limits of probability measures on families of algebraic subvarieties $S^{\perp}$ in $X$ of dimension $2 m=\operatorname{dim} X-\operatorname{dim} S$ and degree $D \rightarrow \infty$ which are eventually transversal/normal to $S$ and which play the role of lipid bilayer rod-shaped molecules; alternatively, 
one may use similar subvarieties in $X^{(r)}$ transversal/normal to the lift $S^{(r)}$ of $S$ to $X^{(r)}$, or else, closed positive $(m, m)$-currents in $X$ transversal/normal to $S$.)

Can one go the other way around and find a (quasi-)functorial gate (arrow) from some classes of partial differential equations $\mathcal{D}$ (and/or geometric structures underlying $\mathcal{D}$ ) to (asymptotically infinite-dimensional) statistical ensembles $\mathcal{S E}$ ? Can one gain in symmetry while passing through such a gate? Is there some asymptotic expansion of (stochastic) perturbations of an extremal $S$ in the space $\mathcal{S E}$ ?

(Possibly, something appears in the physics literature, but I could not trace anything in mathematics. A topological, rather than statistical "gate" of this kind is suggested in [12, but it has a rather limited range of applications.)

What do micelles/liposomes have to do with crystals?

Both can be regarded as low temperature limits of statistical ensembles of particle systems with short range interactions (this is an oversimplification, as we shall see below), where in many (but not all) cases a single particle $M$ can be represented by a (tangent) orthonormal frame in space with the total energy of the ensemble being $\sum_{i j} E\left(M_{i}, M_{j}\right)$ for some binary (pairwise) interaction (potential) energy function $E:$ Frame $\left(\mathbb{R}^{3}\right) \times \operatorname{Frame}\left(\mathbb{R}^{3}\right) \rightarrow \mathbb{R}$ (with the usual contribution of the kinetic energy and the entropy terms).

Also, packings of $S$ by rods are similar to packings of molecules (in $\mathbb{R}^{3}$ ) into crystals. Probably, a "packing of an extremal surface $S$ by rods" is combinatorially periodic on $S$ minus a "small subset" $\Sigma \subset S$ (most likely, $\operatorname{codim} \Sigma=2$ ), provided the minimal packing of the plane by the cross sections of the rods is stably periodic.

Finally, assume the rods are cylindrical and replace them by balls with the same cross sections. The arrangement (or rather a small rearrangement) of these balls along $S$, originally made by the rods, remains extremal but no longer minimizes the energy (due to the excess of positive curvature of the spheres that bound the balls): the Morse index of the corresponding critical point of the energy will be $\approx N$ - the number of rods (or balls) going into making $S$.

This brings liposomal $S$ on equal footing with crystals, as far as the topology is concerned: such surfaces $S$ are associated with certain extremal (not minimal, as for crystals) points of energy on the configuration space of (infinitesimally small) balls. This agrees with the (geometrically) natural (co)homological coupling between spaces of $\mathbb{Z}_{2}$-cycles in Riemannian manifolds $X$ and configuration spaces of balls in $X$ studied by Larry Guth [14. This leaves measure and entropy out of the picture, but, possibly, this can be remedied somehow.

The modification of the energy function, as one passes from rods to balls, is vaguely similar to the multi-parametric/hyperbolic stabilization of generating functions in symplectic Morse theory. Also, Guth's (co)homological coupling can be established between moduli spaces of pseudo-holomorphic curves and (configuration) spaces of disjoint (symplectic images of) balls in symplectic manifolds. This is useful, for instance, for proving parametric symplectic packing inequalities, but the relation between the two symplectic leads remains unclear.

Is there a meaningful picture for extremal arrangements of rods along $S$, where the essential dimension of these rods is strictly between 0 (as is the case for balls in [14]) and codim $S$ (as in [12])?

Are there stochastic extensions/perturbations of the discrete groups in nonEuclidean Lie groups similar to these for micelles and liposomes? 
Is there a symplectization of the stochastic model of micelles and liposomes?

Do subvarieties of algebraic varieties over fields of finite characteristic admit liposome-crystal models?

Water, Hydrogen Bonds, and Hydrophobicity. Biological molecules live in water and interact (e.g., attract one to another) via weak chemical bonds.

The strength of a chemical bond (interaction) is measured by the energy needed to break it, where a convenient reference energy is Boltzmann's $\frac{3}{2} k T$ (where 3 is the dimension of the space and $\frac{1}{2}$ comes from $E=\frac{m v^{2}}{2}$ ) at a room temperature of $T=298^{\circ} \mathrm{K} \approx 25^{\circ} \mathrm{C}$, that is, the average kinetic energy $\frac{m v^{2}}{2}$ of molecules in a liquid (or gas) which is the same for all molecules regardless of their mass $m$. For example, the (square) average speed $v$ of water molecules is about $650 \mathrm{~m} / \mathrm{s}$ at room temperature.

The room temperature $k T$ is close to $2.45 \mathrm{~kJ} / \mathrm{mol} \approx 0.6 \mathrm{kcal} / \mathrm{mol}$ and to $1 / 40 \mathrm{eV}$ in the standard absolute units $(1 \mathrm{kcal} / \mathrm{mol} \approx 4.1840 \mathrm{~kJ} / \mathrm{mol}$ and $\mathrm{eV} \approx 96.5 \mathrm{~kJ} / \mathrm{mol}$, where $1 / \mathrm{mol}=N_{A}^{-1}$ and $N_{A} \approx 6.0221415 \times 10^{23}$ is the Avogadro number - the number of atoms in $12 \mathrm{~g}$ of carbon ${ }^{12} \mathrm{C}$ - by the presently accepted normalization).

For comparison, a green photon carries about $2.5 \mathrm{eV} \approx 100 \mathrm{kT}$ of energy and most covalent bonds have comparable energy: they are stable under room temperature if not exposed to light. Peptide bonds in this sense are unstable in the presence of water. In fact they release about $10 \mathrm{~kJ} / \mathrm{mol} \approx 4 k T$ when they break (by hydrolyzing, i.e., taking back water molecules which they lose when a protein is synthesized). But an easy break at room temperature is prevented by an energy barrier: the half-time for hydrolysis under physiological conditions is a few hundred years [35, 31. (Also the phosphodiester bonds between nucleotide monomers in DNA and RNA are metastable in water.)

Weak chemical bonds in biological molecules are somewhere within 1-10kT (sometimes more), where the strongest among the weak are the so-called H-bonds (hydrogen bonds), which are due to electrostatic attraction between molecules with nonuniformly distributed charges associated with (displacements of) protons of particular hydrogen constituents of molecules.

Bonds close to $k T$ are not static: they constantly break and reappear in thermal equilibrium by exchanging their energy with molecular kinetic energy; also the effective H-bonds between biological molecules $M$ (e.g., amino acids in proteins) are weakened by exchange with H-bonding between $M$ and water molecules.

The H-bonds between water molecules themselves make a complicated dynamic network, where every water molecule can be H-bonded with $n=1,2,3$, 4 other molecules with the average $n$ (estimated by different authors) equal to something between 2.3 and 3.6 at $25^{\circ} \mathrm{C}$. This makes the thermodynamics of water quite peculiar.

For example, the boiling temperature of water $\left(\mathrm{H}_{2} \mathrm{O}\right.$ of atomic weight $\left.\approx 18\right)$ is quite high $\left(100^{\circ} \mathrm{C} \approx 373^{\circ} \mathrm{K}\right)$ compared to other substances of comparable and even larger molecular weight, e.g., $\approx 90^{\circ} \mathrm{K} \approx-183^{\circ} \mathrm{C}$ for $\mathrm{O}_{2}$ of weight $\approx 32$ and $\approx 216^{\circ} \mathrm{K} \approx-57^{\circ} \mathrm{C}$ for $\mathrm{CO}_{2}$ of atomic weight $\approx 44$. Water is still not fully understood on the nano-scale.

Collectively, weak bonds may be quite stable, e.g., folded proteins at room temperature, but the pronounced energy gap between weak and covalent bonds seems essential for the function of biomolecules. 
(The textbook value for the energy of the $\mathrm{H}$-bonds between water molecules is $\approx 5 \mathrm{kcal} / \mathrm{mol}$ but I am not certain if the definition of the energy of an individual $\mathrm{H}$-bond should refer to the ratio $\frac{5}{n} \mathrm{kcal} / \mathrm{mol}$, nor have I followed the experiments for determination of the H-bond energies and free energies, where the concept of the latter I still do not fully grasp.)

Do not think that $k T$ is too small to bother about: if the weak interactions in your proteins went down by $2 \%$, which would amount to a rise of body temperature by $\approx 6^{\circ} \mathrm{K}$, you, above $43^{\circ} \mathrm{C} \approx 316^{\circ} \mathrm{K}$, would be as good as dead.

Yet some thermophilic unicellular organisms (most of them are archaea) strive above the point where all your proteins would unfold. For example, the incredible Strain 121, isolated from a thermal vent deep in the Pacific Ocean, reproduces at $121^{\circ} \mathrm{C}$ and survives for several hours at $130^{\circ} \mathrm{C}$.

But Thermus aquaticus, whose DNA-polymerase is used in commercial PCR for diagnostic DNA amplification, is not an archaeon-it is a thermophilic bacterium.

Hydrophobic molecules, such as (phospho)lipids are not (significantly) polarized and form no H-bonds with water. However, the presence of these molecules in water disrupts $\mathrm{H}$-bonds between water molecules; thus, the whole system tries to minimize the interface between water and a hydrophobic substance.

The resulting surfaces $S$, boundaries of micelles for instance, are not locally minimizing, however, in the class of smooth surfaces, since the water molecules on different sides of $S$ do not (significantly) interact across $S$. The (local) minimality is manifested in discontinuous stochastic perturbations of $S$ in $\mathcal{S E}$. The formation of such an $S$ in $\mathcal{S E}$ does not follow a simple energy gradient curve. It is rather a "gradient tree" something like a branched network of tributaries of (the bed of) a river along which the energy flows downhill, and where the "branches" are physically implemented by disconnected surfaces with boundaries playing the role of nucleating sites ("seeds") in the standard picture of crystal growth (see "crystal growth" in Wikipedia).

All this points to possible stochastic extensions/perturbations of the $\mathrm{vol}^{r}$-model of liposomes and micelles, but the mathematics of this is still nonexistent.

Erythrocytes and Haemoglobin. Erythrocytes carry haemoglobin in the blood of an animal body where they manage to squeeze through 5000nm thin capillaries without major distortion. (Yet, high $\mathrm{pH}$, high calcium concentrations, exposure to glass surfaces, reduced albumin concentrations, and prolonged storage turn erythrocytes into crenated cells, also called burr cells, with short, sharp spikes.)

Haemoglobin is a large (roughly spherical $\approx 6 \mathrm{~nm}$ in diameter) protein built of four structurally similar noncovalently (weakly) bound subunits of 574 amino acids (residues) in total. Each subunit contains a set of alpha-helix segments spatially arranged in a particular globin (supersecondary) pattern, which incorporates a heme group that is an organic molecule with an iron atom in it. This is instrumental for binding oxygen in the lungs, transporting the bound oxygen throughout the body, and carrying $\mathrm{CO}_{2}$ in exchange from the tissues back to the lungs.

The oxygen released from the haemoglobin in muscles binds to myoglobin which stores oxygen in muscles (and also contains for this a heme group with an iron atom).

The binding of oxygen to haemoglobin is a positive cooperative process: when one subunit in haemoglobin becomes oxygenated, it induces a conformation change in the whole protein causing the other three subunits to gain in affinity for oxygen. 
(This is a common trick in nature: use $k$ copies of "something" in order to sharpen an $s$-response to the collective threshold-like $s^{k}$.) Thus, haemoglobin (unlike the single unit myoglobin) switches from binding oxygen in the lungs to its release in muscles where the partial $\mathrm{O}_{2}$-pressure drops $\approx$ twofold. (This "two" is roughly the same as the ratio between sea-level pressure and that at $6 \mathrm{~km}$, above which breathing becomes a problem for humans. Yet, certain birds, e.g., some geese and vultures, fly comfortably above $10 \mathrm{~km}$ at one-quarter of sea-level pressure.)

Haemoglobin makes up about $97 \%$ of the red blood cell's dry content and needs no complicated chemical purification. It was the first protein to be crystallized. The crystals were obtained by Otto Funke (and, perhaps, Karl Reichert) around 1850 by diluting red blood cells with a solvent followed by slow evaporation. (Most pure proteins crystallize only under particular special conditions and/or after a modification of the molecules. The symmetry of the haemoglobin molecule, probably, facilitates crystallization.)

Erythrocytes are continuously produced in the red bone marrow of large bones (in adult humans at the rate $\approx 2.5$ million/second or $\approx 200$ billion/day), and, in mature form (in mammals), they contain no DNA and do not synthesize their proteins. Adult humans have $20-30$ trillion erythrocytes, $\approx 5$ million per cubic millimeter of blood; a human erythrocyte contains about 200-300 million haemoglobin molecules.

All this is just a speck of foam in the sea of biological knowledge. Where does the structure start, and where does it end in this sea?

\section{INFORMATION AND SYMMETRY IN VIRUSES}

In 1956, Crick, Watson, Caspar, and Klug had predicted possible icosahedral symmetry of viruses (such as the herpes simplex virus in the TEM micrograph in Figure 6) by an essentially mathematical reasoning partly based on analysis of X-ray diffraction on crystals of viruses [38, [7].

Why does the random mutation/selection evolutionary mechanism generate $\mathrm{im}$ probable symmetries?

The underlying physical reason for this must be apparent by now: the symmetries of viruses, similarly to crystals, reflect the spacial symmetry of the physical laws as was pointed out in [4, p. 3].

"Self assembly (of a virus) is a process akin to crystallization and is governed by the laws of statistical mechanics. The protein subunits

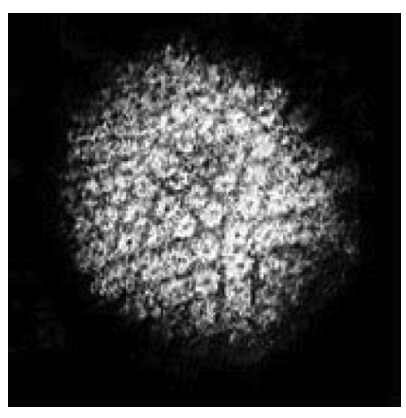

Figure 6. Herpes simplex virus 
and the nucleic acid chain spontaneously come together to form a simple virus particle because this is their lowest (free) energy state."

The symmetry of viral capsids, which has been well established for many viruses (e.g., the viruses of the herpes family are icosahedral $\approx 100 \mathrm{~nm}$ in diameter [2, 37, [3, 17), is no more paradoxical than that of a protein crystal. In a nutshell, if one is ready to disregard the uncomfortable fact that symmetric equations may have nonsymmetric solutions, one might simply say that since the physical world is symmetric, symmetric forms are likely to be functionally as good, if not even better, than nonsymmetric ones. For example, the bilateral symmetry of our bodies is good for walking. (Symmetry is persistent in linear systems, e.g., in small oscillations of viral capsids [1], 36].)

The above geometric/physical consideration shows that viral symmetry is plausible but not necessarily very probable. The decisive reason for the symmetries of viral shells (capsids) set forth by Crick and Watson was that a virus needed to pack "maximum genetic information" in a small shell (capsid) which is built of proteins encoded by the viral genes.

(The idea of DNA codes for proteins was in the air since the 1953 reconstruction of the DNA double helix structure by Crick and Watson, partly based on the X-ray diffraction results of Franklin and Wilkins. Gamov - the author of the Big Bang Theory - suggested in 1954 that each of the 20 amino acids must be coded by a triplet of nucleotides, since $n=3$ is the minimal solution to the inequality $4^{n} \geq 20$, where 4 is the number of different species of nucleotides in DNA. Gamov's is an amazing instance of a simple idea in biology which turned out to be true.)

Indeed, if a virus uses $n$ genes in its DNA (or RNA) say, for the shell proteins, and if each codes for $m$ copies of identical protein molecules, then the resulting viral shell can contain DNA of size $\sim(n m)^{\frac{3}{2}}$. If $m$ is large, this allows smaller $n$ which is advantagous to the virus. (A small virus replicates faster since more copies of it are yielded by an invaded cell.)

The above energy argument implies that the presence of equal copies of protein molecules make symmetric assemblies quite likely if one properly adjusts the " $F-$ angles".

Now, an evolutionary factor enters the game: a symmetric form can be specified by fewer parameters than a functionally comparable nonsymmetric one. For example, a nonsymmetric assembly of molecules may have many different $\vDash$-angles, all of which need to be encoded somehow by the viral DNA (or RNA) while a symmetric form has many of these angles mutually equal. This simplifies Nature's task since it selects from a smaller pool of competing possibilities. (To make true sense of this, one needs to evaluate probabilities of specific molecular/genetic implementations of comparable symmetric and nonsymmetric structures.)

The presence of many identical copies of large heterogeneous "units", e.g., heteropolymeric molecules, is the hallmark of life. These are produced by some universal processes of controlled amplification - another characteristic feature of living systems. The basic instances of this are replication of DNA with the rate up to 1000 (pairs of) nucleotide residues per second, transcribing (by "templating") messenger RNA from DNA ( $\approx 50$ residues per second), and then translation from messenger RNA to proteins by ribosomes ( $\approx 15$ residues per second). On the other hand, ignition of combustion or of a nuclear chain reaction are examples of uncontrolled amplification. 
Abstractly, one minimizes some combination of the total binding energy between protein molecules and the information/selection cost of DNA encoding for these molecules, but a mathematical rendition of the Crick-Watson idea is still pendingnot one of the "isoperimetric animals" cultivated by geometers for the last 3,000 years resembles icosahedral viruses.

At the end of the day, the symmetry of viruses depends on the structural constraints imposed by the geometry of the physical space, which allows the existence of such improbable objects as icosahedra.

(The discovery of regular polyhedra by Neolithic people, who had hardly been inspired by viruses, and a mathematical classification of these polyhedra by Theaetetus, in circa $400 \mathrm{BC}$, may be attributed to the unreasonable mathematical power of the brain's visual processing system. Yet, blind and brainless viruses had discovered icosahedra a couple of billion years earlier.)

\section{Polypeptides And PRoteins: SEQUenCEs, Folds, AND FUnCTions}

A polypeptide is a polymer chain $A_{1} \vDash_{p} A_{2} \vDash_{p} A_{3} \vDash_{p} \cdots$ made out of small basic units-amino acid residues. There are 20 standard amino acids; most (not all) proteins in cells are composed exclusively of these 20. (See "protein" and "amino acid" in Wikipedia.)

A typical length of a chain is 100-300 residues (yet, reaching $>34000$ in titin or connectin, $\mathrm{C}_{132983} \mathrm{H}_{211861} \mathrm{~N}_{36149} \mathrm{O}_{40883} \mathrm{~S}_{69}$ - the adhesion template for the assembly of contractile machinery abundant in skeletal muscle cells).

A residue is what remains of an amino acid after polymerization: the relatively strong covalent peptide bond $\vDash_{p}$ is formed between the carbon atom in each (but the last) amino acid molecule in the chain with the nitrogen atom in the next amino acid with the production of a water molecule.

Immediately upon polymerization, a polypeptide chain, synthesized in the cell by the ribosomes and their "helpers" (making, conceivably, the most complicated chemical system in the astronomically observable universe) folds into a specific rather compact shape, called a protein, held by additional weak binding forces between residues, mainly by hydrophobicity "pressure" and hydrogen bonds. (Seven out of 20 amino acids, e.g., tryptophan and cysteine, are rather hydrophobic. They tend to conglomerate in the protein hydrophobic core with small exposure to the surrounding water.)

Some proteins are made of several polypeptide chains. For example, haemoglobin is composed of four $\approx 150$-long subunit chains.

The beauty of proteins comes as much from a multitude of "little structures" within and around particular species of them as from yet unknown but vaguely felt general mathematical principles which underly their existence and properties. A thousand papers are dedicated to these "little ones"; e.g., haemoglobin boasts $>10^{6}$ entries in Google, and RuBisCO, the most abundant protein found on Earth, appears on > 250,000 pages of Google. (Much of this was explained to me by Arthur Lesk and Chen Keaser.)

Amino acids are small molecules, about $5 \AA$ in diameter $\left(1 \AA=0.1 \mathrm{~nm}=10^{-10} \mathrm{~m}\right)$, where 18 out of 20 standard amino acids are composed of carbon, nitrogen, oxygen, hydrogen and the remaining two (cysteine and methionine) also contain sulfur. The smallest amino acid, glycine, has 10 atoms in it and the largest, tryptophan has 27 atoms. 


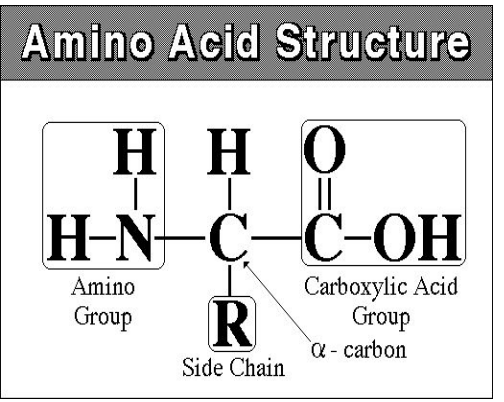

Figure 7. Amino acid
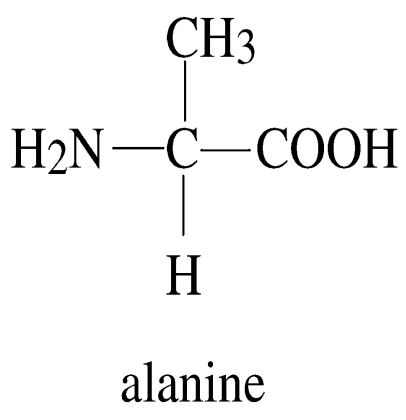

Figure 8. Alanine<smiles>O=C(O)C1CCCN1</smiles>

proline

Figure 9. Proline

Each amino acid contains the main chain of nine atoms $\mathrm{H}_{2} \mathrm{~N}-\mathrm{CH}-\mathrm{C}-\mathrm{O}_{2} \mathrm{H}$ and a side chain (R-group) covalently bound to the central carbon atom called $\mathrm{C}_{\alpha}$ (or $\mathrm{C}^{\alpha}$ ) in the main chain. As an exception, proline has its 5-cyclic side chain also bound to N. See Figures 7, 8, and 9,

Fifteen amino acid have tree-like side chains, e.g., single $\mathrm{H}$ for $\mathrm{R}$ in (marginally hydrophobic) glycine and $\mathrm{CH}_{3}$ in (mildly hydrophobic) alanine, while four have single cycles (with covalent bonds for the edges) in them, e.g., proline. The largest, tryptophan, has two cycles.

Formally, a protein, or rather a polypeptide at this stage, is represented by a long word written on the backbone (which is a linear graph or a string with the peptide bonds for edges) in the letters of labeled graphs - the side chains of amino acid residues. See Figures 10 and 11 . 
<smiles>NCC(=O)O</smiles>

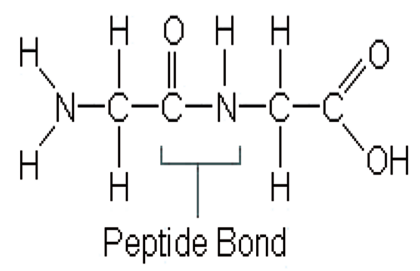

A molecule of water is removed from two glycine amino acids to form a peptide bond.

Figure 10. Peptide bond

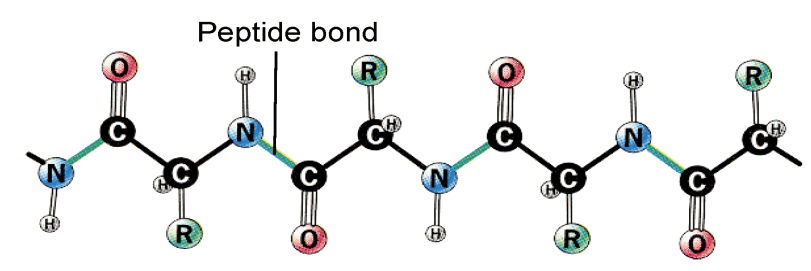

Figure 11. Polypeptide

The linear structure of proteins was suggested in 1902 by Franz Hofmeister and, a few hours later at the same meeting, by Emil Fischer, but many doubted that peptide bonds were strong enough to hold such long molecules together in the presence of thermal agitations. (On the surface of things, titin, for example, must have a half life of a few days. Is it more stable in conformation? Is it being constantly recycled? Does its function need stability? I have not looked enough into the literature.)

The beginning of the "era of sequencing" is landmarked with the determination of the primary/sequential structure of the two polypeptide chains of insulin by Frederick Sanger in 1955 followed by Sanger's method for sequencing RNA and DNA in 1970s. (This, in mathematics, can be compared to the turn in algebraic geometry and topology marked by the work by Jean Pierre Serre in the 1950s.)

(Insulin is a very small messenger protein produced in the pancreas of animals, secreted to blood and controlling the intake of glucose by cells by binding to receptors on the cell membrane. It consists of two polypeptide chains of 21 and 30 residues held together by weak forces further stabilized by three covalent S-S bonds between sulfur atoms in the side chains of cysteine residues. There are two Cysteines in the 30-chain and four in the 21-chain; one S-S bond joins two residue in the latter, and two bonds bridge the two chains together. Insulin is produced in two stages: first a cell synthesizes an insulin precursor, a polypeptide chain of $84=21+$ $30+33$ residues, and later the 33 -segment is excised by a specific protein-cleaving enzyme.) 


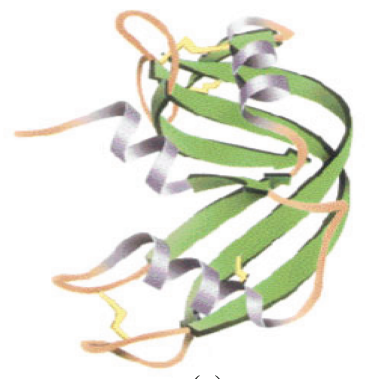

(a)

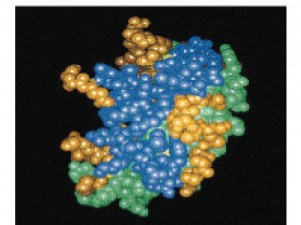

(b)

FiguRE 12. Bovine pancreatic ribonuclease-A

The backbone chain of atoms in the polypeptide chain (with all but some terminal $\mathrm{O}$ and $\mathrm{H}$ omitted) looks as follows:

$$
\mathrm{H}_{2} \mathrm{~N}-\mathrm{C}^{\alpha}-\mathrm{C}^{\prime} \vDash_{p} \mathrm{~N}-\mathrm{C}^{\alpha}-\mathrm{C}^{\prime} \vDash_{p} \mathrm{~N}-\mathrm{C}^{\alpha}-\mathrm{C}^{\prime} \vDash_{p} \cdots \vDash_{p} \mathrm{~N}-\mathrm{C}^{\alpha}-\mathrm{C}^{\prime} \vDash_{p} \mathrm{~N}-\mathrm{C}^{\alpha}-\mathrm{CO}_{2} \mathrm{H} \text {. }
$$

The peptide bonds $\vDash_{p}$ are rather rigid with planar angles of approximately $120^{\circ}$. The spatial flexibility of a polypeptide is mainly due to rotational freedom around $\mathrm{N}-\mathrm{C}^{\alpha}$ and $\mathrm{C}^{\alpha}-\mathrm{C}^{\prime}$ bonds in each residue $A$, where the subset $R_{A}$ in the 2-torus $\mathbb{T}^{2}$ of possible rotational angles, limited by spatial constraints of the side chain of $A$, is called the Ramachandran domain (plot).

Thus, the spatial conformation of the backbone of a chain $A_{1} \vDash_{p} A_{2} \vDash_{p} \cdots \vDash_{p} A_{n}$ is representable by a point in (or a probability measure on) the Cartesian product of $n$ Ramachandran domains, $R_{A_{1}} \times R_{A_{2}} \times \cdots \times R_{A_{n}} \subset \mathbb{T}^{2 n}$, while a full conformation of a polypeptide depends on extra degrees of rotational freedom around some bonds in the side chains.

Most native (i.e., coming from living organisms) polypeptides assume, under specified conditions, a unique (if it is generously understood) spacial conformation, where some atoms, which may be far apart in the chain, come close together due to weak interactions (bonds) between them. Besides noncovalent weak bonds, the stability of some protein (e.g., of insulin), ribonucleases, and many snake poisons, is reinforced by covalent S-S bridges between sulfur atoms in cysteines.

Bovine pancreatic ribonuclease- $A$ (atomically $\mathrm{C}_{575} \mathrm{H}_{907} \mathrm{~N}_{171} \mathrm{O}_{192} \mathrm{~S}_{12}$, which is represented in Figure 12 schematically and atomically) is a common enzyme in a biologist's lab. It uses 19 out of 20 amino acids, with eight cysteines involved in four S-S bridges and four methionine residues containing sulfur atoms; only tryptophan is missing. It has three $\alpha$-helices and three $\beta$-hairpins, where two of them form a four-stranded antiparallel $\beta$-sheet (defined below).

It is secreted by a cow's pancreas; its RNA cleaving activity is stable under up to $100^{\circ} \mathrm{C}$, mainly due to the reinforcement of the enzyme structure by the S-S bonds. It is involved in digestion of RNA produced by micro-organisms residing in bovine stomachs, where cellulose is broken down by symbiotic bacteria and protozoa.

Its amino acid sequence of 124 residues was determined in 1960, and the 3-D structure was solved in 1967 by X-ray diffraction analysis.

It is commonly accepted by biologists that all information required to specify the correct three-dimensional conformation of a protein is contained in its primary amino acid sequence. (The mathematical interpretations of these "all", "information", "required", "correct", etc., are by no means unique.) 
In bacterial cells, proteins should fold within at most a few minutes, since the life cycle of many bacteria is about 20 minutes.

Many (possibly most) proteins of lengths up to 150-250, when they are artificially unfolded by heating or by disturbing weak interactions with some chemical agent, spontaneously fold back (sometimes as fast as in a few milliseconds) to the native state when the conditions return to normal.

(It is often emphasized that folding in cells is a co-translational process: some proteins spontaneously fold but some may have a hard time folding if they start from a random position in the configuration space and/or will be not in contact with ribosomes and/or other protein complexes accompanying translation.)

This was established by Anfinsen's team in 1961 for bovine ribonuclease-A, where the reappearance of BRA's ability to degrade RNA was used as a witness of proper folding.

The (properly understood) uniqueness of foldings agrees with the existence of the crystal forms for many proteins since a heterogeneous mixture of nanoparticles is unlikely to make a crystal. Yet, there is some controversy about the universality, uniqueness, and mechanisms of folding.

(Ambiguities and inconsistencies in presentation of "basic facts" in biology are frustrating to a mathematician. For example, you can find statements in the literature that insulin cannot renaturate - to refold if unfolded - but some authors claim that the 33-chain is not crucial for bringing two other chains together and that under proper renaturation conditions the native insulin is obtained from a scrambled one with $25 \%$ yield that increases to $75 \%$ if the two chains are covalently linked. But frustration turns into joy if you think of many interpretations of ambiguous statements allowing a variety of mathematical developments.)

How proteins fold. The basic folding patterns of proteins are called the secondary structures which are divided into two groups: $\alpha$-helices and $\beta$-sheets; both are associated with the $\mathbb{Z}$-symmetry of the proteins' backbones.

Helical structure was conjectured by William Astbury in the early 1930s on the basis of changes in the X-ray fiber diffraction of moist wool or hair fibers upon stretching; a detailed atomic model was worked out by Pauling, Corey, and Branson in the early 1950s.

(An essential component in the wool and hair, as well as in the outermost layer of cells of human skin, fingernails, and in birds' feathers, is keratin. Keratins make a group of fibrous proteins with helical molecules twisting around each other with many S-S bridges between cysteine residues resulting in rigid structures. Human hair is about $15 \%$ cysteine. The characteristic smell of burning hair is due to the high presence of sulfur in there.)

A typical helix contains about ten amino acids (about three turns) but some may have over forty residues. Helices are represented by rigid rods in schematic pictures of proteins. See Figure 13 .

Although the helix is formed by H-bonding between the backbone residues, different amino acid sequences have different propensities for forming an $\alpha$ helical structure.

(For example, proline does not fit into helices because it misses an $\mathrm{H}$-atom at $\mathrm{N}$ needed for the H-bond (with an $\mathrm{O}$ ), while glycine, the smallest amino acid, disrupts helices, apparently, because its Ramachandran domain $R_{\text {glyc }} \subset \mathbb{T}^{2}$ is quite large, making fixed angles in a helical arrangement "entropically expensive".) 

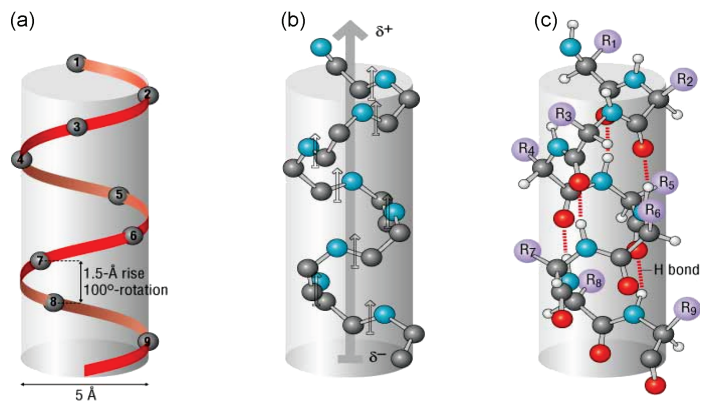

Figure 13. Protein helices

The $\beta$-sheet, the second form of regular secondary structure in proteins, displays $\mathbb{Z} \oplus \mathbb{Z}$-symmetry. Such a sheet consists of several parallel or antiparallel (with respect to the $\mathrm{H}_{2} \mathrm{~N} \rightarrow \cdots \rightarrow \mathrm{CO}_{2} \mathrm{H}$ direction of the backbone chain) $\beta$-strands, typically $5-10$ amino acids long, connected laterally by $\mathrm{H}$-bonds and forming a pleated (often twisted) sheet. An example of (antiparallel) $\beta$ is a hairpin turn (pictured in Figure 14) where the two $\beta$-strands (almost) follow each other on the backbone.

(The $\beta$-linkages between strands of different polypeptide chains often make insoluble aggregates - amyloids, e.g., in Alzheimer's and mad cow diseases, where the crystalline-like structure of amyloids is not well understood.)

The combinatorial structure defined by $\beta$-sheets is much richer than what comes from $\alpha$-helices: the arrangement of strands into sheets is a kind of transformation

(a)

native strand alignment

ype I turn

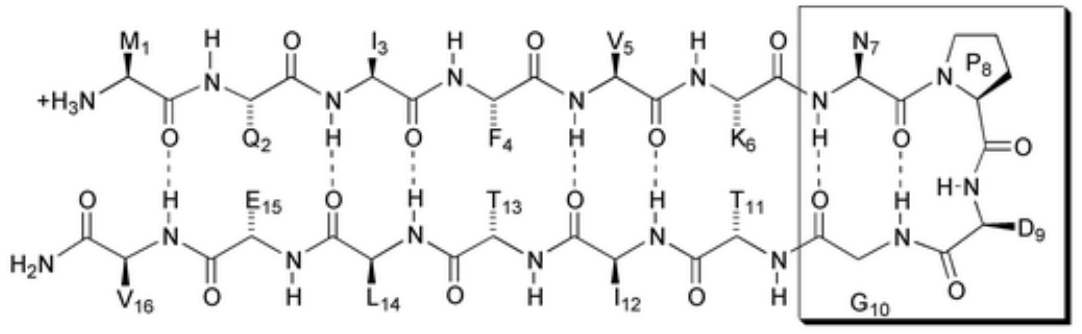

(b) non-native strand alignment

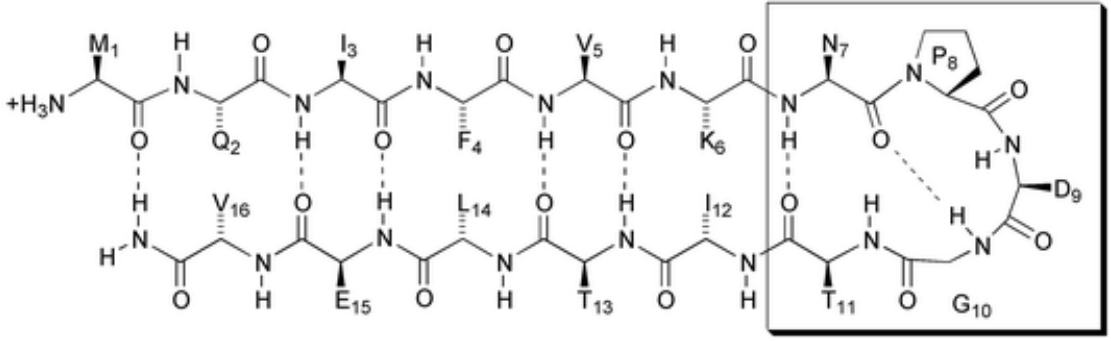

FiguRE 14. Hairpin turn 
of the $\mathbb{Z}$-structure of the backbone to $\mathbb{Z} \oplus \mathbb{Z}$ of the sheets. The combinatorics of this transformation is determined by a subset $\mathcal{S}$ of particular segments $S$ in the backbone, which represent the strands, and a graph on the vertex set $\mathcal{S}$ where the edges join the neighbor strands $S$ in the sheets and where, moreover, the edges are labeled by the types of these neighbor relations, e.g., being parallel or antiparallel.

The combinatorics of this graph (which is by itself not very informative) combined with the order structure on the strands in the backbone displays, in many instances [5], form tree-like (nested) patterns similar to those in parsing of sentences into words and phrases in context free languages.

Besides pure combinatorics, the rigid rods (for $\alpha$-helices) and plates (for $\beta$-sheets), joined by rather flexible loops, make a particular arrangement (of the backbone of a protein) in the 3 -space, called the super-secondary/tertiary structure of a protein 22.

One still lacks a comprehensive formal language for describing such structures, as was emphatically pointed out to me by Arthur Lesk on several occasions. (See [30] for a new mathematical approach.)

Protein binding. Most of the functions of proteins in cells depend on the specific binding of a protein $P$ to another molecule $M$ or to a particular class of molecules.

For instance, several protein molecules make a viral capsid (shell) by binding one to another.

Signaling/messenger proteins, e.g., insulin, bind to specific receptor proteins on the exposed membranes of cells.

Regulatory proteins bind to specific, a few nucleotide pairs long, segments of DNA, thus enhancing or suppressing the transcription of RNA.

Octamers of (for types of core) histone proteins make spools around which DNA winds with $\approx 150$ nucleotide pairs per turn (additionally stabilized by linker histones). The 30nm-thick necklace of spools on DNA eventually assembles into a few micrometers long chromosome in a (eukaryotic) cell. (The length of human DNA is $\approx 2 \mathrm{~m}$ per cell - a couple of light days - about ten times the distance from the sun to Pluto-for the totality of cells in the body.)

Proteins are rather sticky due to the nonuniformity of electric charges on their ragged surfaces, and they tend to nonspecifically bind one to another. Amazingly, this does not disrupt the normal activity of the cell. (A particular shape of the charge distribution can turn the total Coulomb force between two particles into an attraction, where the particles may be neutral or even carry nonzero charges of the same sign, e.g., both negatively charged.)

Catalysis. Certain proteins, called enzymes, speed up chemical reactions 9]. (About 4,000 such reactions are known in cells.) For example, let $M$ be a molecule where some specific bond is metastable: the energy is released when this bond is broken, such as the peptide bond in the water environment or the phosphodiester bond in RNA between nucleotides.

What prevents this break from happening spontaneously, or rather makes it highly improbable, is a potential barrier - a "mountain range" in the configuration space $Y$ of $M$ around a metastable state $y_{0} \in Y$ which prevents the flow of energy from $y_{0}$ - a local minimum of the energy function on $Y$ - to the true minimum $y_{\text {min }}$ of the energy. 
A catalyst provides a channel across this barrier (or widens an existent but very narrow and/or long twisted channel) which allows a passage from $y_{0}$ to $y_{\min }$. This may be implemented by different chemical mechanisms (which are still not fully understood [18]).

Digestive enzymes break down polymeric macromolecules. For example, pepsin released in the stomach and trypsin produced in the pancreas break peptide bonds in proteins at certain amino acid residues which are specific for particular enzymes, while ribonucleases bind to RNA molecules at specific places and cleave the covalent bonds between the nucleotide residues.

Catalase, which is contained in tissues of organisms exposed to oxygen, e.g., in your saliva, splits $\mathrm{H}_{2} \mathrm{O}_{2}$ into water and oxygen. This is an instance of a kinetically perfect enzyme limited only by the diffusion rate of the substrate, where each molecule of catalase decomposes millions of $\mathrm{H}_{2} \mathrm{O}_{2}$ molecules per second. (If you spit into a water solution of $\mathrm{H}_{2} \mathrm{O}_{2}$, the liquid starts bubbling with oxygen.)

What are the configuration space representations depicting the catalytic functions of these enzymes?

Enzymes are extremely good at what they do: some speed up reactions $10^{17}$ $10^{18}$ fold. (This is said about orotidine $5^{\prime}$-phosphate decarboxylase 26. Notice that an experimental measurement of the rate of such a noncatalized reaction $R$ must be experimentally quite different from measuring this $R$ accelerated 100000000000000000 times.)

But $\mathrm{RuBisCO}$, which catalyzes the commonest chemical reaction in green leaves and algae - the rate limiting step of photosynthetic $\mathrm{CO}_{2}$ fixation is surprisingly inefficient-it "fixes" $1-5$ molecules of $\mathrm{CO}_{2}$ per second [11, 34].

\section{ENERGY LANDSCAPES AND PROTEIN PROBLEMS}

Let $X=X(P)$ be the configuration space of a given polypeptide chain $P$ in $\mathbb{R}^{3}$, and let $E: X \rightarrow \mathbb{R}$ be the total energy "summing up" the weak interactions between the (atoms in the) residues.

Can one find the "ground state" $x_{\min }=x_{\text {fold }}$ which minimizes the energy and corresponds to the folded protein? (After all, if Nature does it in a few seconds, why can a mathematician not do it?)

The folding process in this $X(P)$-model corresponds to the downstream gradient flow of $E$ where the orbit of every $x \in X$ eventually arrives at $x_{\text {min }}$. But $E$ may have lots of local minima: their number is likely to grow exponentially with the dimension of $X$ that is (roughly) proportional to the number $N$ of residues in $P$. If so, a protein cannot fold reasonably fast, if at all, for most $E$.

The problem of shallow local minima disappears in a more realistic model where thermal fluctuations are incorporated into the picture (and, thus, the loss of entropy under transition of the disordered polypeptide chain to a compact conformation is taken into account). Now, the folding process is represented by a random walk which is biased according to $E$, where the probability density of a point $x_{1} \in X$ moving in time $\Delta t$ to a nearby point $x_{2}$ is proportional to $\Delta t \exp \left(E\left(x_{1}\right)-E\left(x_{2}\right)\right)$. (Roughly, this corresponds to smoothing the function $E$, thus, erasing insignificant local minima.)

The ground/folded state is represented in the randomized flow by a "small" neighbourhhod $U_{\text {fold }}$ of $x_{\min }=x_{\text {fold }}$ such that our random walk stays in $U_{\text {fold }}$ most of the time, i.e., with probability $\geq 1-\varepsilon$ for a (moderately) small $\varepsilon>0$. 
The domain $U_{\text {fold }}$, apparently, may be rather large for some proteins: the positions of certain residues in loops (unstructured segments in the chain) may be statistically significantly (i.e., for not very small $\varepsilon$ ) distributed over distances $d$ comparable to the size $D$ of the protein, say with $d>0.1-0.2 D$. Also, a comprehensive model must incorporate the statistical dynamics of water molecules, which is, in particular, crucial for the hydrophobic component of the folding force.

Yet, one may expect that a typical $E$ would have many local minima with rather wide/deep attraction basins, so that $x$ would keep jumping from one $U$ to another. In other words, the sublevel $U(1-\varepsilon)=E^{-1}(-\infty, x=x(\varepsilon)) \subset X$, such that the stationary probability measure $\mu_{s t}(U(1-\varepsilon))$ equals $1-\varepsilon$, may have many connected components spread over $X$ and not localized at all around any $x_{\text {fold }}$, and even if $U(\varepsilon)$ is localized, the time of arrival to it from a random $x \in X$ may be too long. There must be something special about $E$ which allows (fast) folding.

It is hard to say more as we do not have a sufficient understanding of the connectivity properties of sublevels (not to mention their higher-dimensional homologies, which is an interesting problem in its own right) of such random functions on highdimensional spaces. Something in this regard is provided by percolation theory, but this concerns limits of spaces of fixed dimension, where the size of a domain goes to infinity (e.g., in lattices) or, conversely, where only the dimension goes to infinity (as for the $n$-cliques and $n$-cubes).

Here, however, both the size of the space $X=X(P)$ as well as its dimension grow with the length $N$ of $P$, and where something interesting happens not so much in the limit but at specific (large but not very large) values of the parameters. (Too long polypeptide chains, well above 500 residues, do not fold, if at all, into anything like compact protein globules, but some large proteins may consist of several independently folded globular domains while others, such as titin and keratin, have a fibrous structure.)

Apparently, the backbone degrees of freedom contribute to the size of $X$ as well as to its dimension, while the side chains' degrees of freedom contribute only to $\operatorname{dim} X$.

If the size of $X$ is small on the oscillation scale of $E$ (which is controlled, to a large extent, by the average value of the gradient of $E$ ) and if the dimension of $X$ is large, one expects a unique large cluster (connected component) of a (low energy) sublevel which covers most of (the measure of) $X$. (Yet, beware of something such as $E\left(x_{1}, \ldots, x_{i}, \ldots, x_{n}\right)=\sum_{i} E_{i}\left(x_{i}\right)$, where the number of local $\mathrm{m}$ inima is $N_{\min }(E)=\prod_{i} N_{\min }\left(E_{i}\right)$.)

But if $X$ is large compared to $\operatorname{dim} X$, then one expects many local minima and high disconnectedness of sublevels (of comparable energy) of random functions $E$.

In the latter case one cannot expect folding of generic polypeptides, but one can imagine that some special polypeptides fold similarly to how crystals and micelles are formed - the folding process is directed by something like a riverbed with tributaries in $X=X(P)$ which channels the gradient flow toward $X_{\text {fold }}$, where specific patterns in (the combinatorics of tributaries of) this riverbed, say $R=R(P)$, correspond to interactions between particular important (groups of) residues in $P$ and where these patterns were selected by Nature for native proteins $P$ in the course of evolution [39], 43], 42], 20]. (In the case of protein crystals, the role of selector is taken by a crystallographer.)

Can one make this mathematically precise? 
Can one get an insight into $R$ for specific (families of) proteins on the basis of their conformations and/or of their biochemical properties?

Can one artificially design proteins which would fold by controlling their $R$ ?

A more realistic mathematical problem is that of finding a class of models of highdimensional stochastic gradient-like systems which may be far from real proteins but where the above questions have positive answers.

But should one stick to this huge space $X(P)$ anyway? After all, one has no experimental access to all of $X$ (even the theoretical existence of such "full $X$ " is debatable) but rather to some quotients of $X$ corresponding (to sets of) particular observables (functions) on this space, where the random dynamics on $X$ defined by the probabilities of transitions from one domain in $X$ to another within an infinitesimal time interval $\delta t$ naturally induces such dynamics on every quotient space $Y$ of $X$. (This suggests that some projective limit of the observable quotients of $X$ may replace $X$.)

In particular, the connectivity of sublevels of a function $E$ on a topological space $X$ is encoded by such a quotient- the sublevel tree $T=T(X, E)$. This is a tree with a continuous map $\tau: X \rightarrow T$ and a function $E_{T}: T \rightarrow \mathbb{R}$, such that $E=E_{t} \circ \tau$ and where the map induced by $\tau$ from the set of connected components of every $t$-sublevel of $E$ (i.e., of the subset $\left.E^{-1}(-\infty, t] \subset X\right)$ onto the $t$-sublevel set for $E_{T}$ is one-to-one.

The tree $T$ comes with a natural metric on it for which $E_{T}: T \rightarrow \mathbb{R}$ is isometric on every edge of $T$. Besides, it carries the stationary measure of the random walk induced on it from $X$.

(Actually, every measure on $X$ induces a measure on $T$. It is tempting to use the product of the angular measure of rotations around simple covalent bonds in the residues in $P$, but it is unclear to me if this makes much sense.)

How much of protein properties, and of molecular assemblies in general, can be expressed in the language of these trees $T$ ?

There is a natural convolution product operation on such trees (which is again a tree), say $T_{1} \star T_{2}$, which corresponds to noninteracting systems, where $T_{1} \star T_{2}$ equals the sublevel tree for the sum of energies, $E_{1}+E_{2}$ on $T_{1} \times T_{2}$. (This generalizes/refines the convolution of measures on $\mathbb{R}$, where a somewhat different kind of object-a general graph instead of a tree - comes up if one uses levels instead of sublevels of E.)

If the systems do interact, then the full energy on the product of the respective spaces is written as $E_{1}+E_{2}+E_{1,2}$, where the interaction term $E_{1,2}$ is usually localized on a certain relatively small part of the product space.

For example, the effect of a catalyst, e.g., an enzyme, can be, apparently, seen in terms of this $E_{1,2}$ on the products of full configuration spaces but the $T$-quotients may be too small for this.

What are the smallest quotients of the full configuration spaces which would allow an adequate geometric description of enzymatic catalysis? How much does this depend on the type of an enzyme $P$ ?

The above mathematical problems are compounded by physical ones [10]:

1. The weak interaction energies between (atoms in) residues are known only approximately: their quantum mechanical derivation is far beyond our computational capability and no direct experiment can determine interatomic/molecular interaction with sufficient precision. 
2. The total interaction energy $E$ of a polypeptide is not the sum of the pairwise residue interaction energies.

However, even if nonbinary and nonstrictly additive, the interaction energy is a relatively simple (unknown) function of (the sequential composition of) a protein (in a given solvent), which, probably, can be encoded with a reasonable accuracy by something like $10^{4}-10^{6}$ bits of information. (For example, if the energy were the sum over the pairwise residue interaction energies, $E=\sum E_{i j}$, which we wanted to estimate up to an $\varepsilon$, we would need about $20^{2} \log (N / \varepsilon)$ bits for all proteins with $N$ amino acid residues.) On the other hand, one has much high throughput experimental data on (properties of) proteins, where each experiment carries at least one bit.

What is a general mathematical parameter fitting method(s), which, when applied to proteins, could provide (an effective version of) the total inter-residue interaction energies?

(Such an approach is pursued in bioinformatics [39, [43, [42, but it does not seem to incorporate the biochemical data available, e.g., on the calorimetry of protein (un)foldings and/or on protein-protein' binding, say, for proteinimmunoglobulin.)

What percentage of polypeptide chains fold? The number of, say, $N$-long amino acid sequences is $20^{N} \approx 10^{1.3 N}$, but the overwhelming majority of polypeptide chains, probably, do not make anything even vaguely resembling proteins. (Random sequences of letters make no sentences in the English language either.)

But is there any way to describe the subset (space) $\mathcal{P}$ of all conceivable proteins $P$ in the full sequence space? What is the cardinality of this space $\mathcal{P}$ ?

Write the cardinality of the set of conceivable protein sequences of length $N$ as $20^{\sigma N}$ for $0<\sigma<1$ and think of $1-\sigma$ as codimension (coentropy) of the space $\mathcal{P}$, where the dimension of the space of all sequences is normalized to 1 . In other words, $1-\sigma$ represents the number of equations or constraints which a sequence has to satisfy in order for $P$ to behave "protein-like", where the propensity to fold is an essential ingredient of being "protein-like".

In reality, $\sigma$ depends on $N$ as well as on a particular protein $P$-a point in $\mathcal{P}$ where $\sigma$ is evaluated.

The full space $\mathcal{P}$ is too large to be studied experimentally, but one can evaluate the proportion of proteins $P^{\prime}$ obtained from a given $P$ by a few mutations, e.g., by substitutions of some residue by another one in the sequence, such that $P^{\prime}$ still folds.

A lower bound on such local $\sigma(P)$ for native proteins can be extracted from the data on the mutation rate of proteins estimated by comparing sequences of homologous proteins of different organisms [29, 41. Namely, let $r=r(P)$ be the mutation rate of $P$ and $R=R(P)$ be the rate of fictitious mutations, usually called synonymous mutations of DNA which do not change the corresponding amino acid (because of the redundancy of the genetic code). Then the ratio $r / R$ provides a plausible lower bound on $\sigma$, since the mutations must not only preserve the folding but also the functionality of $P$.

The evolutionary data suggest that the (folding component of the) value of $\sigma$ is somewhere around $1 / 2$ (rather than being close to 0 or to 1 ; overlaps of some viral genes suggest $\sigma>2 / 3$ for small proteins), but it remains unclear how much a particular value of $r / R$, which greatly varies across different families of proteins, depends on the type of folding (e.g., on the super-secondary structure of a protein $P$ ) 
and how much is due to the functional constraints. (Possibly, one may extract some information by comparing the mutation rates of proteins with a similar structure versus such rates for proteins with similar functions.)

For example, a protein which specifically interacts with several neighbor proteins is expected to have a small ratio $r / R$ but this does not necessarily affect the folding component of its $\sigma$. This ratio is sometimes effectively zero, e.g., for the histone protein $\mathrm{H} 4(\approx 100$ amino acid residues, essentially identical for humans and rodents separated by $\approx 100$ million years of evolution $[33$ ), and then the meaning of $r / R$ becomes questionable.

(This histone's conservatism, apparently, depends on a finely tuned structural association with neighboring proteins and DNA, as well as on specific, not fully understood, histones' functions, which are more complicated, for example, than those of highly mutable proteins in viral capsids.)

Interestingly enough, already in 1904, George Henry Falkiner Nuttall observed that rabbits' antibodies/immunoglobulins developed against human blood proteins did equally well at precipitating the blood serum of African apes (but not so of Asian apes), thus showing a close similarity of the corresponding proteins prior to even any idea of their sequences. Then comparative immunology was used by Morris Goodman in 1961 for establishing evolutionary relationships among primates.

However, there is no general (semi-mathematical) approach combining biochemical and sequential/evolutionary data for evaluation of essential structural properties of proteins such as the relative roles of structural and functional constraints reflected in $r / R$. (But the evolutionary comparison is systematically used in bioinformatics for predicting protein conformations by their sequences.)

One may expect that the main contribution to $\sigma$ can be expressed by pairwise correlations between residues at specific positions on the chain which somehow influence one another in the conformation but proving (and even stating) this mathematically seems hard; one is tempted to look at similar more approachable models of design and/or evolution of stochastic gradient flows of functions on high-dimensional spaces. (Probably, there are tractable model problems in the percolation theory.)

Everything we presented in this paper hardly scratches the surface of what is known (and unknown) about crystals, cell membranes, virus capsids and proteins, where understanding the structure and molecular function of proteins constitutes the first step in the solution of the main biological problem of the sequencing era.

Describe the arrow genotype $\leadsto$ phenotype, where genotype is given by the genome of an organism, possibly "ornamented" by some epigenetic data (e.g., methylation of some bases and positions of some regulatory proteins on DNA).

This problem can be divided into several parts.

1. Determine the domain of definition of the map " $\rightarrow$ " by finding a realistic (mathematical) description of the subset $\mathcal{G}$ of sequences which may serve as viable genomes $G$, of "conceivable organisms", where this description must be expressed in the language of sequences.

There may be several such descriptions on different levels of precision, where such a description is supposed to be only approximate with a balance between the degree of approximation/precision and mathematical simplicity/complexity of the description.

We have briefly addressed this for individual proteins $P$, where a protein sequence and function is determined (modulo alternative splicing, translational regulation, and post-translational modifications) by the DNA code for $P$. 
2. Formally describe "phenotype", but let it be only approximate, on several levels of precision.

This is nontrivial even for an individual protein $P$ where its phenotype includes both the structure (conformation) of $P$ as well as its function(s) and where the simplest to describe (but often hard to determine) among functions are the protein's binding and enzymatic properties.

3. Represent (possibly, only approximately/statistically) the "space of phenotypes" or, rather, a significant (sub)quotient of this space, as a quotient of the space of (possibly, slightly annotated) genomes by some equivalence relation with an effective description of this relation in the sequential language. (This is similar to describing the real world as a quotient space of the "space of sentences" of a natural language.)

In particular, define and evaluate some numerical measure(s) of "redundancy of the map $\sim$ " associated to the cardinalities of the fibers of this map.

A special and a more realistic subproblem is doing this in a neighborhood of an individual native protein $P$, where this redundancy may be expressed by an equivalence relation on the set of amino acid sequences close to those of $P$ and giving a protein $P^{\prime}$ similar to $P$. In particular, we want to say something interesting on the domain of continuity of the map $\sim$ and on jumps of $\sim$ at the discontinuity points.

A mathematician's role in solving these problems may consist in designing a parameter-fitting scheme for determination of a mathematically/logically simple(st) arrow $\leadsto$ (or fragments of $\leadsto$ ) compatible with (constrained by) two kinds of data:

A. The data (e.g., obtainable from protein data banks) on sequences, structures and functions of proteins;

B. The known physics/chemistry of proteins. (These data need a preliminary uniform formal representation.)

Besides the above, there are two other general problems in molecular biology with a mathematical tint to them: combinatorial design of high throughput experiments and description of the moduli space(s) of proteins (and genomes).

The latter is associated to the map from the protein tree of life into the protein space $\mathcal{P}$, where the slow dynamics of evolution shapes the fast dynamics of folding and of enzymatic catalysis. (The tree of life is not a "bare tree": the horizontal gene transfer, artificial construction of chimeric proteins, the position and the number of genes on DNA which code a protein, protein interactions, etc., add much extra structure to this tree.)

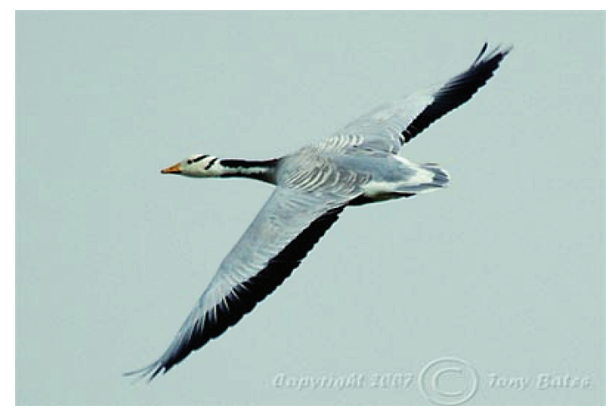

Figure 15. Bar-headed goose 


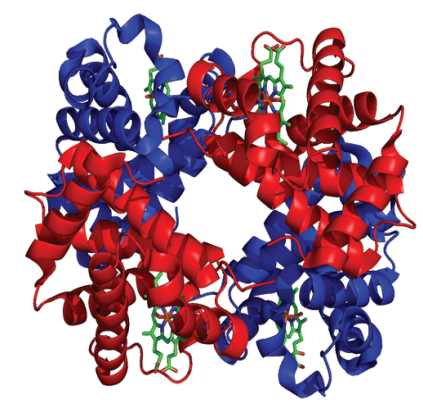

FiguRE 16. Haemoglobin molecule

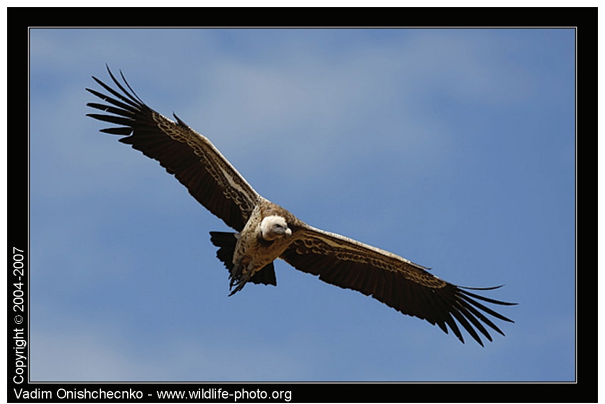

FiguRE 17. Rüppell's vulture

The bar-headed goose (see Figure 15) flies up to $10 \mathrm{~km}$ when migrating over the Himalayas, covering up to 1000 miles in one day aided by high altitude jet streams. Its haemoglobin allows the goose to breathe $-50^{\circ} \mathrm{C}$ air at $25 \%$ sea-level density. This haemoglobin differs from the haemoglobin of its lowland relative by four amino acids, where, arguably, only one of the substitutions, proline $\mapsto$ alanine, contributes to the jump in the goose's haemoglobin oxygen affinity and its ability to fly high. See Figure 16 .

Understandably/regretably, our present ideas on "protein problems" fall into a traditional mathematical framework. It is hoped that an attempt to solve them may lead us to something new and unexpected.

The goose has two kinds of haemoglobin in its blood, where only one of them has the elevated oxygen affinity; the presence of the second one allows the goose's adaptation to low altitudes.

Even more remarkably, Rüppell's vulture, (G. rueppellii) has four different types of haemoglobin in its blood [23. See Figure 17.

(On November 29, 1973, an aircraft collided with a bird over Côte d'Ivoire at an altitude of $11,300 \mathrm{~m}$ as was recorded by the pilot shortly after the impact. The plane landed safely at Abijan. The remnants of the bird allowed their identification as that of G. rueppellii 6 .)

\section{About the AUthor}

Misha Gromov is a professor at Institut des Hautes Études Scientifiques, Paris, France, and Courant Institute of Mathematical Sciences, New York. 


\section{REFERENCES}

[1] S. Andersson, K. Larsson, M. Larsson, Virus Symmetry and Dynamics, http://www. sandforsk.se/sandforsk-articles/all.all.articles.htm.

[2] A.J. Cann, Principles of molecular virology; 4th ed. Amsterdam: Elsevier Academic Press, 2005.

[3] S. Casjens, Principles of virion structure, function and assembly, and J.S. Baker and J.E. Johnson, Principles of virus structure determination in Structural Biology of Viruses, W. Chu R.M. Burnett, R.L. Garcia, Wah Chiu, (eds.), 1997; Oxford University Press.

[4] D. L. D. Caspar, A. Klug, (1962) "Physical Principles in the Construction of Regular Viruses" Cold Spring Harbor Symposia on Quantitative Biology XXVII, Cold Spring Harbor Laboratory, New York. pp. 1-24.

[5] Y.S. Chiang, T.I. Gelfand, A.E. Kister, I.M. Gelfand, New classification of supersecondary structures of sandwich-like proteins uncovers strict patterns of strand assemblage. Proteins, 2007.

[6] Collision between a Vulture and an Aircraft. THE WILSON BULLETIN December, 1974, Vol. 86, No. 4, http://elibrary.unm.edu/sora/Wilson/v086n04/p0461-p0462.pdf.

[7] Conformational Proteomics of Macromolecular Architecture: Approaching the Structure of Large Molecular Assemblies and Their Mechanisms of Action (with CD-Rom) (Paperback) by R. Holland Cheng (Author), Lena Hammar (Editor), 2004, a historical survey by Morgan.

[8] H. R. Crane, Principles and problems of biological growth. The Scientific Monthly, Volume 70, Issue 6, pp. 376-389 (1950). (This article is often referred to but I could not see it since it is not freely available on the web.)

[9] Alan Fersht, Structure and Mechanism in Protein Science: A Guide to Enzyme Catalysis and Protein Folding, W.H. Freeman \& Co Ltd; 3rd Revised edition, 1998.

[10] A. V. Finkelstein, O. B. Ptitsyn, Protein Physics: A Course of Lectures. Academic Press, 2002.

[11] Howard Griffiths, Designs on Rubisco, Nature 441, 940-941 (22 June 2006).

[12] M. Gromov, Isoperimetry of waists and concentration of maps, Geom. Funct. Anal. 13 (2003), 178-215. 178-215. MR1978494 (2004m:53073)

[13] M. Gromov, Mendelian Dynamics and Sturtevant's Paradigm. In Geometric and Probabilistic Structures in Contemporary Mathematics Series: Dynamics, (Keith Burns, Dmitry Dolgopyat, and Yakov Pesin, editors), American Mathematical Society, Providence RI (2007).

[14] Larry Guth, Minimax problems related to cup powers and Steenrod squares, Geom. Funct. Anal. 18 (2009), 1917-1987. MR 2491695 (2010e:53071)

[15] W. Helfrich, Z. Naturforsch, Bending energy of vesicle membranes: General expressions for the first, second, and third variation of the shape energy and applications to spheres and cylinders, Phys. Rev. A 39, 5280 - 5288 (1989).

[16] Mike Howard, Introduction to Crystallography and Mineral Crystal Systems. http://www.rockhounds.com/rockshop/xtal/index.html.

[17] John E. Johnson, Virus assembly and maturation, in Folding and Self-assembly of Biological Macromolecules, E. Westhof, and N. Hardy, editors, World Scientific, 2004.

[18] Kenneth E. NeetDagger, Enzyme Catalytic Power Minireview Series, J. Biol. Chem., Vol. 273, Issue 40, 25527-25528, October 2, 1998.

[19] A. Klug, The tobacco mosaic virus particle: structure and assembly. Philos. Trans. R. Soc. Lond. B Biol. Sci. March 29, 1999, 354(1383): 531-535.

[20] Rachel Kolodny, Patrice Koehl, Leonidas Guibas and Michael Levitt, Small Libraries of Protein Fragments Model Native Protein Structures Accurately, J. Mol. Biol. (2002) 323, 297-307.

[21] D.J. Kushner, Self-Assembly of Biological Structures, Bacteriol Rev. 33:302-345. 1969

[22] Arthur M. Lesk, Introduction to Protein Architecture Oxford University Press, 2000.

[23] Wen-Hsiung Li, Molecular Evolution, Sinauer Associates, Sunderland, MA, USA. 1997.

[24] D.B. Lukatsky, E.I. Shakhnovich, Statistically enhanced promiscuity of structurally correlated patterns. Physical Review E 77 (2) 020901, 2008; and arxiv.org/pdf/q-bio/0603017.

[25] Klaus Mainzer, Symmetries of Nature, Walter De Gruyter, New York, 1996. MR953086 (89i:00033)

[26] B.G. Miller, R. Wolfenden, "Catalytic proficiency: the unusual case of OMP decarboxylase.". Annual Rev. Biochem. 71 (2002), 847-885. 
[27] Masayoshi Nakasako, Water-protein interactions from high-resolution protein crystallography. Philos. Trans. R. Soc. Lond. B Biol. Sci. August 29, 2004; 359(1448): 1191-1206.

[28] Christo N. Nanev, How do crystal lattice contacts reveal protein crystallization mechanism? Cryst. Res. Technol. 43, No. 9, 914-920 (2008).

[29] Laszlo Patthy, Protein Evolution, Blackwell Science, 1999.

[30] R. C. Penner, Michael Knudsen, Carsten Wiuf, Joergen Ellegaard Andersen, Fatgraph Models of Proteins, eprint arXiv:0902.1025.

[31] A. Radzicka, R. Wolfenden, Rates of uncatalyzed peptide bond hydrolysis in neutral solution and the transition state affinities of proteases, J. Amer. Chem. Soc., 1996, Vol. 118 (No.26)

[32] K. K. Reddi, Tobacco mosaic virus with emphasis on the events within the host cell following infection, Advances in Virus Research, Volume 17, Kenneth Manley Smith ed., 1972, Science.

[33] Alejandro P. Rooney and Masatoshi Nei, Purifying Selection and Birth-and-death Evolution in the Histone H4 Gene Family. Molecular Biology and Evolution 19:689-697 (2002).

[34] F. Robert Tabita, Thomas E. Hanson, Huiying Li, Sriram Satagopan Jaya Singh and Sum Chan, Function, Structure, and Evolution of the RubisCO-Like Proteins and Their RubisCO Homologs, Microbiol. Mol. Biol. Rev. December 2007; 71(4): 576-599.

[35] Bernard Testa, Joachim M. Mayer, Hydrolysis in Drug and Prodrug Metabolism: Chemistry, Biochemistry, and Enzymology, Science, 2003.

[36] G. A. Vliegenthart, G. Gompper, Mechanical deformation of spherical viruses with icosahedral symmetry, Biophys. J., August 1, 2006; 91(3): 834-841.

[37] Virology, http : //pathmicro.med.sc.edu/mhunt/intro - vir.htm. Virus Structure, http : //www.microbiologybytes.com/introduction/structure.html. Principles of Virus Architecture, http://web.uct.ac.za/depts/mmi/stannard/virarch.html.

[38] History of Structural Virology, http://virologyhistory.wustl.edu/timeline.htm.

[39] David Baker lab, http://depts.washington.edu/bakerpg/.

[40] Andrzej Kolinski group, http://biocomp.chem.uw.edu.pl/.

[41] Eugene Koonin group, http://www.ncbi.nlm.nih.gov/CBBresearch/Koonin/.

[42] Olivier Lichtarge lab, http://mammoth.bcm.tmc.edu/.

[43] Rama Ranganathan lab, http://www.hhmi.swmed.edu/Labs/rr/.

[44] Eugene Shakhnovich group, http://www - shakh.harvard.edu/research/index.html.

[45] Jeffrey Skolnick group, http://cssb.biology.gatech.edu/skolnick/.

Institut des Hautes Études Scientifiques, Paris, France; and Courant Institute of Mathematical Sciences, New York 